\title{
The Effect of Spirituality on Quality of Life of Patients with Chronic Kidney Disease and Its Correlation with the Mental Health Status and Cognitive Perception for Their Illness. Study Protocol
}

\author{
Evangelos Fradelos $^{{ }^{*}}$, Foteini Tzavella ${ }^{1}$, Eumorfia Koukia ${ }^{2}$, Sofia Zyga ${ }^{1}$ \\ ${ }^{1}$ Department of Nursing, Faculty of Human Movement and Quality of Life, University of Peloponnese, Sparta, \\ Greece \\ ${ }^{2}$ Faculty of Nursing, University of Athens, Athens, Greece \\ Email: evagelosfradelos@hotmail.com, ${ }^{*}$ efradelos@med.uoa.gr
}

Received 2 August 2015; accepted 20 August 2015; published 25 August 2015

Copyright (C) 2015 by authors and OALib.

This work is licensed under the Creative Commons Attribution International License (CC BY). http://creativecommons.org/licenses/by/4.0/

(c) (i) Open Access

\section{Abstract}

Background: Patients who suffer from chronic renal disease face problems in many aspects of their life, problems that are physical, social as well as mental, such as stress, anxiety, depression. Patients with Chronic Kidney Disease (CKD) exhibit a number of spiritual needs, which relate to and influence the psychological adaptation to the illness. Aim: The aim of the present study is to investigate spirituality and spiritual needs of patients with CKD and the possible relation between spirituality, quality of life, mental health and perceptions of their illness. Moreover, the possible differences in spirituality, mental health, Quality of Life (QoL) and perceptions for the disease among End Stage Renal Disease (ESRD) patients and patients suffering by third and fourth stage CKD will be assessed. Method: 300 patients diagnosed with $3^{\text {rd }}, 4^{\text {th }}$ stage and ESRD undergoing Hemodialysis (HD) treatment will be recruited to participate in this study. Each subject's spiritual wellbeing, QoL, mental health status and illness perception will be measured using the following instruments: 1) The Functional Assessment of Chronic Illness Therapy-Spiritual Well-Being Scale (FACIT-Sp-12), 2) The Depression, Anxiety, Stress Scale-DASS-21, 3) The Illness Perception Questionnaire, 4) The Quality of Life Index SF-36. Results: Patients' spiritual well-being is expected to be related to their $Q o L$, mental health and illness perception.

\section{Keywords}

CKD Patient, Spiritual Wellbeing, Quality of Life, Mental Health, Illness Perceptions

${ }^{*}$ Corresponding author.

How to cite this paper: Fradelos, E., Tzavella, F., Koukia, E. and Zyga, S. (2015) The Effect of Spirituality on Quality of Life of Patients with Chronic Kidney Disease and Its Correlation with the Mental Health Status and Cognitive Perception for Their Illness. Study Protocol. Open Access Library Journal, 2: e1783. http://dx.doi.org/10.4236/oalib.1101783 


\section{Introduction}

Renal failure or Renal disease (RD) is a medical condition in which the kidneys fail to adequately filter waste products from the blood or to perform the regulating operations. Thus, products that are normally excreted in the urine are accumulated in the organism and they deregulate the endocrine and metabolic function, and cause disturbances in fluids, electrolytes and acid-base balance. Chronic Kidney Disease (CKD) is a condition that is characterized by gradual and progressive, generally not reversible reduction of renal function, which is caused by damage of the kidneys, with varied causes. Chronic Kidney Disease is defined as kidney damage with glomerular filtration rate (GFR) lower than $60 \mathrm{~mL} / \mathrm{min} / 1.73 \mathrm{~m}^{2}$ for three months or longer. There are 5 stages of CKD, based on the glomerular filtration rate (GFR) values, which are measured directly or calculated mathematically from the serum creatinine values. Nowadays, CKD is recognized as a serious problem of public health and the world wide prevalence is estimated to be within the range of $8 \%-16 \%$ [1] [2]. According to the World Health Organization (WHO), when patients reach a GFR of less than 15\%, they are classified into the end-stage renal disease (ESRD) group. This stage is characterized by the necessity of hemodialysis or kidney transplantation so that the patients can be kept alive [3].

Patients who suffer from chronic kidney disease face problems in many aspects of their life, such as physical, social as well as mental problems, such as stress, anxiety and depression [4]-[8]. Most patients that suffer from ESRD depend on health services for kidney replacement therapies such as hemodialysis. Moreover, they are obliged to adapt to new data and experience enormous changes in their daily habits. Restrictions not only in food and liquid intake must be enforced but also in various activities and functions [9] [10]. The management of illness presupposes various changes in patients' life [11]-[13].

During the past few years, an intense research interest for spirituality and its possible relation with the physical and mental health has been observed, mainly in the chronic diseases as well as in life threatening situations. A lot of studies on spirituality have heart, cancer and AIDS patients as population targets. Many of these studies have led to the conclusion that the spiritual beliefs of people are positively related with health, longevity and rehabilitation of physical diseases [14]-[16].

Studies on patients with CKD regarding spirituality have led to the conclusion that these patients exhibit a number of spiritual needs, which relate to and influence the psychological adaptation to the illness. At the same time, these studies showed that the development of a close therapeutic relationship between nurses and patients in renal departments is influenced by the positive experiences of these patients' spiritual care [17]-[19].

\section{Background}

The relation between spirituality and health has been recorded since antiquity. In ancient civilization, the healer and the religious leader was often the same person. Thus, this same person would care for both body and spirit at the same time [14]. For many individuals spirituality and religion are important dimensions of their existence and they are a source of support that contributes to the well-being and the confrontation of difficulties of daily life. For many patients the incorporation of spiritual beliefs in the healing process is of vital importance and it has proved to be related with positive results for mental health [15]. The positive influence that spirituality can have in the patients' perception for their health, but also in coping with and adaptation to a serious and life threating illness has been documented by many researches. Specifically, studies that were conducted on patients with diagnosis of serious disease, like cancer in advanced stage, led to the conclusion that spirituality is an important supportive factor that influences both the adaptation to the illness, and the general situation of patients' mental health [20] [21].

A comparative study among healthy and chronically suffering people that was conducted by Sodhi and Manju [21], showed that there is a statistically significant positive correlation among various factors of spirituality and positive mental state. The individuals with a high level of spirituality present higher indicators of mental health. The researchers report that someone can positively strengthen the health of an individual by providing a therapeutic environment that improves the spiritual beliefs and this way, a positive attitude toward life is also 
achieved. Spiritual beliefs and practices can be important coping resources and positive adaptation to a chronic illness, and can contribute to the mental health of people that suffer from a chronic disease [21].

Moreover, studies have been conducted to examine the positive relation between spirituality and immune system [22]. The action mechanism that is presented in these studies is about the effect of spirituality in the improvement of stress control, which affects the immune system. Spirituality provides mechanisms for better confrontation with stressful situations, rich social support and intensification of personal values [22].

The attendance of patients who suffer from terminal cancer with low life expectancy, in religious and spiritual practices appears to decrease the likelihood of depression and melancholy occurrence [23]. At the same time, a study investigating the relation between spirituality and pain, showed that spirituality is a pain coping strategy, but was not found to correlate with the intensity of pain that the patients experience [23]. Despite this, the same researchers propose that further research must be conducted, so that the clinical usefulness of the incorporation of religious and spiritual practices is assessed, specifically in palliative care [23].

Regarding chronic kidney disease, the passage to the end stage and the beginning of kidney replacement treatment, constitutes an important landmark in the whole course of the patient. The changes in everyday routine and functionality of these patients are important and radical. Specifically, the individual is rendered as a "patient”, two or three times a week with his life depending on the blood dialysis machine and on the people that maintain and handle it. In this phase of kidney replacement treatment the social and mental support are important parameters of care. Comparative studies between patients with CKD who are in pre-dialysis stage and patients who are in kidney replacement treatment, have been conducted. These studies mainly investigate healthrelated quality of life (QoL), which appears to be considerably different between these two groups [4] [8] [24]. However, studies which investigate the spirituality and its effect in the two groups of patients, do not appear to exist.

Contemporary health care services models that take into consideration all the aspects of patient individuality and the needs that derive from them, mental, spiritual and social, have been found to considerably contribute to the adaptation of the patient to the chronic illness and to the better rehabilitation. The recognition of spiritual needs and their fulfillment are of vital importance, while their contribution is presented as very important in the multidimensional care of patients with chronic renal illness. The effects that the spiritual distress has on a mental and social level, but also on the result and adaptation to the illness, make the conduct of further research necessary with reliable tools of evaluating the patients' spiritual needs and the repercussions that they have in the general condition of the patient [15] [17] [18] [25].

\section{Aim}

The aim of the present study is to investigate spirituality and spiritual needs of patients with CKD and the possible relation between spirituality, quality of life, mental health and perception for their illness. Moreover, the possible differences in spirituality, mental health, quality of life and perception for the disease among ESRD patients and patients suffering from third and fourth stage CKD will be assessed.

\section{Methodology}

A cross sectional study will be conducted using a structured questionnaire on a convenience sample. The research sample will be 300 patients that suffer from CKD of stage 3, 4 and ESRD. According to Nomogram by Altman [26] for statistical significance level alfa $=5 \%$, a statistical power of $95 \%$ and standardized effect size 0.59 the necessary sample size is $n=150$ (150 dialysis patients and 150 pre dialysis patients).

The inclusion criteria are: 1) >18 years old, 2) ability to speak and read Greek, 3) to have been diagnosed with CKD in stage 3, 4 or ESRD, 4) haemodialysis (HD) treatment for at least 6 months for the ESRD patients, 5) adequate time and space orientation.

The exclusion criteria are: 1) patients suffering from psychiatric or cognitive disorders, 2) patients with functional disabilities, visual or hearing disorders.

Written approval to conduct the study will be sought from the Scientific Council of the hospitals. According to the ethical standards of the Helsinki Declaration all patients will be informed about their rights to refuse or stop the participation in the study. To respect the rules of research ethics, the patients will be asked to complete anonymous questionnaires. 


\subsection{Data Collection}

Preparations for recruitment of the participants, measurements will start in winter of 2016 and will be completed within 2016. Data will be obtained by anonymous self-administrated questionnaires consisting of the following instruments.

\subsection{Instruments}

The psychometric tools included in the study are presented below.

1) A semi-structured questionnaire concerning demographic data (age, gender, employment, education).

2) The Functional Assessment of Chronic Illness Therapy-Spiritual Well-Being Scale (FACIT-Sp-12) [27] [28].

It is a scale created by Cella et al. (1993) and has been widely used for the assessment of spirituality in chronic patients. It constitutes a part of a bigger evaluation tool that measures important factors of functionality in patients with chronic illness. Specifically, it includes three subscales: the meaning of life, peace and sense of support and strength that is drawn from faith. Each factor of spirituality includes 4 questions of the five-level Likert scale with the 0 representing "not at all” and the 4 corresponding to "a lot”. The questions refer to the last 7 days. Greater scores represent greater spiritual well-being. The total sum of all answers gives information about the general spiritual well-being. It is a valid tool with a high reliability index (Cronbach’s alpha 0.87) [27] [28].

3) The Depression, Anxiety, Stress Scale-DASS-21 [29].

It is a 21 questions scale that includes three subscales: depression, anxiety and stress. Each subscale includes 7 questions of the five-level Likert scale from 0 to 4 . The 0 corresponds to "not stated for me" and the 4 corresponds to "it absolutely expresses me". In order to calculate each single subscale score but also the general score, the score of the corresponding questions is being added and multiplied by two. The English version of DASS-21 is a reliable assessment tool with Cronbach's alpha index 0.90, 0.95 and 0.93, respectively, for depression, anxiety and stress and 0.97 for the total grades. In this study the Greek version of the questionnaire will be used, which has a reliability index of 0.96 [29]-[31].

4) The Illness Perception Questionnaire (Brief Version) [32].

It is a 9 item scale, which came out of a synopsis of an initial scale of 100 questions and it measures perception of patients for their disease. From the 9 questions, the 5 assess the cognitive perception of patient for their disease, 2 the emotional perception, 1 assesses the patient's comprehension degree of disease and, finally, 1 asks the patient to report what they consider to be the causes of their illness. All the questions are marked from 0 to 10 , apart from the ninth, which is open and asks the patient to describe which they consider to be the main causes of their illness. High scores in the 8 questions show negative perception for the illness. It is a valid tool with high reliability index (Cronbach's alpha 0.91) [32]. In this study the Greek version of the questionnaire will be used [33].

5) The Quality of Life Index SF-36 [34].

It is an internationally recognized valid and reliable tool for assessing the quality of life and it has been translated in many languages. It consists of 36 questions that are distributed in eight subscales: physical function, role function, physical pain, general health, vitality, social functioning, emotional well-being and mental health. The first four factors are the part of the questionnaire that is about the Physical Health and the last four factors are about the Mental Health. In this study the Greek version of the questionnaire will be used which has a reliability index Cronbach's alpha $>0.70$ [35].

\section{Statistical Analysis}

The data statistical analysis will be performed with the use of the statistical packet SPSS version 21. After the coding of responses, data processing will be done with descriptive and inferential statistical analysis methods (namely, analysis of variance, correlation and regression). The level of statistical significance will be set to $\mathrm{p}<$ 0.05 .

\section{Discussion}

This study will examine the possible correlation between spiritual well-being and QoL, the relationship between 
spirituality and mental health. Moreover, the effect of spiritual wellbeing in the illness perception will be assessed. The findings of this study can be used in order to determine the spiritual needs of chronic kidney patients, their impacts on QoL, mental health status and the cognitive perception of their illness.

\section{Conclusion}

Chronic kidney disease is a very complex condition with multiple complications not only on a physical but also on a mental and social level. Thus a holistic care approach is necessary [36]. The successful completion of this study will shed light on the effect of spirituality on quality of life of patients with chronic kidney disease and its correlation with the mental health status and cognitive perception of their illness in CKD patients. Thus, it can help nurses and clinicians with the management of chronic kidney disease and to develop ad hoc interventions promoting spiritual wellbeing.

\section{Limitations}

During the conduction of the study the following difficulties are expected to arise:

1) interference of external factors such as noise, fatigue of the patients, interruptions by the personnel as the study will take place in hospital environment.

2) low health literacy of the patients. The researcher will read the medical terms of the questionnaires and explain them.

\section{References}

[1] NKF.K/DOQI (2002) Clinical Practice Guidelines for Chronic Kidney Disease: Evaluation, Classification, and Stratification. American Journal of Kidney Diseases, 9, S1-S266.

[2] Jha, V., Garcia-Garcia, G., Iseki, K., et al. (2013) Chronic Kidney Disease: Global Dimension and Perspectives. Lancet, 382, 260-272. http://dx.doi.org/10.1016/S0140-6736(13)60687-X

[3] Schieppati, A. and Remuzzi, G. (2005) Chronic Renal Disease as a Public Health Problem: Epidemiology, Social, and Economic Implications. Kidney International Supplements, 68, 7-10. http://dx.doi.org/10.1111/j.1523-1755.2005.09801.x

[4] Finkelstein, O.F., Wuerth, D. and Finkelstein, H.S. (2009) Health Related Quality of Life and the CKD Patient: Challenges for the Nephrology Community. Kidney International, 76, 946-952. http://dx.doi.org/10.1038/ki.2009.307

[5] Theofilou, P. (2012) The Relation of Social Support to Mental Health and Locus of Control in Chronic Kidney Disease. Journal of Renal Nursing, 4, 18-22. http://dx.doi.org/10.12968/jorn.2012.4.1.18

[6] Theofilou, P. (2013) Association of Insomnia Symptoms with Kidney Disease Quality of Life Reported by Patients on Maintenance Dialysis. Psychology, Health \& Medicine, 18, 70-78. http://dx.doi.org/10.1080/13548506.2012.674144

[7] Theofilou, P., Aroni, A., Tsironi, M. and Zyga, S. (2013) Measuring Pain Self-Efficacy and Health Related Quality of Life among Hemodialysis Patients in Greece: A Cross-Sectional Study. Health Psychology Research, 1, e30, 160-161. http://dx.doi.org/10.4081/hpr.2013.e30

[8] Theophilou, P. (2010) Psychiatric Disorders in Chronic Periodic Hemodialysis. Rostrum of Asclepius, 9, 420-440.

[9] Gerogianni, S., Babatsikou, F., Gerogianni, G,. Grapsa, E., Vasilopoulos, G., Zyga, S. and Koutis, Ch. (2014) Concerns of Patients on Dialysis: A Research Study. Health Science Journal, 8, 423-437.

[10] Tsiamis, G., Alikari, V., Fradelos, E., Papapetrou, S. and Zyga, S. (2015) Assessment of Quality of Life and Fatigue among Haemodialysis Patients. American Journal of Nursing Science. Special Issue: Mental Health Care: Aspects, Challenges and Perspectives, 4, 66-73.

[11] De Santo, G.N., Perna, A., El Matri, A., De Santo, M.R. and Cirillo, M. (2010) Survival Is Not Enough. Journal of Renal Nutrition, 22, 211-219. http://dx.doi.org/10.1053/j.jrn.2011.10.010

[12] Eckardt, K., Coresh, J., Devuyst, O., Johnson, J.R., Köttgen, A., Levey, S.A. and Levin, A. (2013) Evolving Importance of Kidney Disease: From Subspecialty to Global Health Burden. The Lancet, 382, 158-169. http://dx.doi.org/10.1016/S0140-6736(13)60439-0

[13] Loghman, M.A. (2013) Medication Noncompliance in Patients with Chronic Disease: Issues in Dialysis and Renal Transplantation. American Journal of Managed Care, 9, 155-171.

[14] Martsolf, D.S. and Mickley, J.R. (1998) The Concept of Spirituality in Nursing Theories: Differing World-Views and Extent of Focus. Journal of Advanced Nursing, 27, 294-303. http://dx.doi.org/10.1046/j.1365-2648.1998.00519.x

[15] Greenberg, D. (2003) Spirituality in Medicine: Does It Fit? Penn Pulse, 99, 1-7. 
[16] Zullig, K.J., Ward, R.M. and Horn, T. (2009) The Association between Perceived Spirituality, Religiosity and Life Satisfaction: The Mediating Role of Self-Rated Health. Social Indicators Research, 79, 255-274. http://dx.doi.org/10.1007/s11205-005-4127-5

[17] Davison, N.S. and Jhangri, S.G. (2010) Existential and Supportive Care Needs among Patients with Chronic Kidney Disease. Journal of Pain and Symptom Management, 40, 838-843. http://dx.doi.org/10.1016/j.jpainsymman.2010.03.015

[18] Davison, N.S. and Jhangri, S.G. (2013) The Relationship between Spirituality, Psychological Adjustment to Illness, and Health-Related Quality of Life in Patients with Advanced Chronic Kidney Disease. Journal of Pain and Symptom Management, 45, 170-178. http://dx.doi.org/10.1016/j.jpainsymman.2012.02.019

[19] Deal, B. and Grassley, S.J. (2012) The Lived Experience of Giving Spiritual Care: Phenomenological Study of Nephrology Nurses Working in Acute and Chronic Hemodialysis Settings. Nephrology Nursing Journal, 39, 471-496.

[20] Lin, H.-R. and Bauer-Wu, S.M. (2003) Psycho-Spiritual Well-Being in Patients with Advanced Cancer: An Integrative Review of the Literature. Journal of Advances Nursing, 44, 69-80. http://dx.doi.org/10.1046/j.1365-2648.2003.02768.x

[21] Sodhi, R. and Manju, D.R. (2013) Spiritually and Mental Health among Normal and Chronic Disease Group. International Journal of Research Studies in Psychology, 2, 59-68. http://dx.doi.org/10.5861/ijrsp.2012.106

[22] Koenig, H.G., Cohen, H.J., George, L.K., Hays, J.C., Larson, D.B. and Blazer, D.G. (1997) Attendance at Religious Services, Interleukin-6, and Other Biological Parameters of Immune Function in Older Adults. International Journal of Psychiatry in Medicine, 27, 233-250. http://dx.doi.org/10.2190/40NF-Q9Y2-0GG7-4WH6

[23] Yates, J.W., Chalmer, B.J., St James, P., Follansbee, M. and McKegney, F.P. (1981) Religion in Patients with Advanced Cancer. Medical and Pediatric Oncology, 9, 121-128. http://dx.doi.org/10.1002/mpo.2950090204

[24] Avramovic, M. and Stefanovic, S. (2012) Health-Related Quality of Life in Different Stages of Renal Failure. Artificial Organs, 36, 581-589. http://dx.doi.org/10.1111/j.1525-1594.2011.01429.x

[25] Doenges, M.E., Moorhouse, M., Frances, M. and Alice, C. (2009) Deployment Guide in Nursing Care Plan. Pashalidis Publications, Athens.

[26] Altman, G.D. (1991) Practical Statistics for Medical Research. Chapman \& Hill, London.

[27] Cella, D.F., Tulsky, D.S., Gray, G., Sarafian, B., Linn, E., Bonomi, A., et al. (1993) The Functional Assessment of Cancer Therapy Scale: Development and Validation of the General Measure. Journal of Clinical Oncology, 11, 570579.

[28] Peterman, A.H., Fitchett, G., Brady, M.J., Hernandez, L. and Cella, D. (2002) Measuring Spiritual Well-Being in People with Cancer: The Functional Assessment of Chronic Illness Therapy—Spiritual Well-Being Scale (FACIT-Sp). Annals of Behavioral Medicine, 24, 49-58. http://dx.doi.org/10.1207/S15324796ABM2401_06

[29] Lovibond, S.H. and Lovibond, P.F. (1995) Manual for the Depression Anxiety Stress Scales. 2nd Edition, Psychology Foundation, Sydney.

[30] Lyrakos, G.N., Arvaniti, C., Smyrnioti, M. and Kostopanagiotou, G. (2011) Translation and Validation Study of the Depression Anxiety Stress Scale in the Greek General Population and in a Psychiatric Patient's Sample. European Psychiatry, 26, 1731. http://dx.doi.org/10.1016/S0924-9338(11)73435-6

[31] Henry, J.D. and Crawford, J.R. (1995) The 21-Item Version of the Depression Anxiety Stress Scales (DASS-21): Normative Data and Psychometric Evaluation in a Large Non-Clinical Sample. British Journal of Clinical Psychology, 44, 227-239. http://dx.doi.org/10.1348/014466505X29657

[32] Broadbent, E., Petrie, K.J., Main, J. and Weinman, J. (2006) The Brief Illness Perception Questionnaire. Journal of Psychosomatic Research, 60, 631-637. http://dx.doi.org/10.1016/j.jpsychores.2005.10.020

[33] Karademas, C.E., Bakouli, A., Bastounis, A., Kallergi, F., Tamtami, P. and Theofilou, M. (2008) Illness Perceptions, Illness-Related Problems, Subjective Health and the Role of Perceived Primal Threat: Preliminary Findings. Journal of Health Psychology, 13, 1021-1029. http://dx.doi.org/10.1177/1359105308097967

[34] Ware, J.E. and Sherbourne, C.D. (1992) The MOS 36-Item Short-Form Health Survey (SF-36). I. Conceptual Framework and Item Selection. Medical Care, 30, 473-483. http://dx.doi.org/10.1097/00005650-199206000-00002

[35] Anagnostopoulos, F., Niakas, D. and Pappa, E. (2005) Construct Validation of the Greek SF-36. Health Survey Quality of Life Research, 8, 1959-1965. http://dx.doi.org/10.1007/s11136-005-3866-8

[36] Alikari, V., Matziou, V., Tsironi, M., Theofilou, P. and Zyga, S. (2015) The Effect of Nursing Counseling on Improving Knowledge, Adherence to Treatment and Quality of Life of Patients Undergoing Hemodialysis. International Journal of Caring Sciences, 8, 514-518. 


\section{Abbreviations}

RD: Renal Disease

CKD: Chronic Kidney Disease

GFR: Glomerular Filtration Rate

WHO: World Health Organization

ESRD: End-Stage Renal Disease

QoL: Quality of Life

HD: Haemodialysis 